Conclusion Early surgical intervention ( $\leq 5$ days) was associated with a shorter duration of hospital stay and antibiotic therapy as well as a lower complication rate. We recommend that corrective surgery be undertaken as soon as reasonably possible.

\section{OCCIPITAL LOBE EPILEPSY IN KUWAITI CHILDREN: A STUDY OVER 2 YEARS}

doi:10.1136/archdischild-2012-302724.1498

MZ Chinnathambi, SK Cheruveettara, A Moosa. Pediatrics, Adan Hospital, Kuwait, Kuwait

Objective To study the clinical profile and therapeutic outcome of occipital lobe epilepsy in Kuwaiti children attending epilepsy clinic in a regional hospital.

Design A retrospective case series study over two years.

Setting Analysis of case files from the epilepsy clinic of a regional hospital who were diagnosed with occipital epilepsy between Aug 2009 to Aug 2011

Subjects Eleven cases were retrieved for study.

Results Ten children had the late childhood form of Gastaut (age 6-12 years) and one had the early childhood form of Panayiotopoulos (age 3 years). Ten children had visual symptoms all of whom presented with sudden onset and cessation of blindness lasting for seconds to few minutes and 5 had visual hallucinations. Half of them had headache. Ten were idiopathic and one of them had focal occipital encephalomalacia. The EEG showed occipital spikes in 8 cases, extraoccipital spikes in 2 cases and generalized discharges in 1 case. All the patients responded well to antiepileptic drugs (carbamazepine, oxcarbazepine, sodium valproate, leviteracetam).

Conclusion Occipital epilepsy, though rare must be considered in children with paroxysms of transient visual symptoms with or without head ache and must be distinguished from migraine.

\section{STUDY OF THE PATTERN OF CEREBRAL PALSY AMONG CHILDREN IN ALEXANDRIA, EGYPT}

doi:10.1136/archdischild-2012-302724.1499

HH Abdeldayem, F Kamel, M Khalil, S Elmansy. Pediatrics, Pediatric Neurology Unit, Faculty of Medicine, Alexandria University, Alexandria, Egypt

Cerebral Palsy (CP) is the commonest neuromotor impairment in childhood. In recent years, there has been mounting changes in $\mathrm{CP}$ pattern. Advances in perinatal and newborn cares have been responsible for these changes. Advances in medical techniques have been also responsible for the extension of the life of CP children to an age of diagnosis and classification. Over the last several years, our knowledge about CP has been substantially improved, but much works still remains. Despite of its importance, the problem is largely ignored in developing countries including Egypt. The aim of our study was arranged for:

1. Giving a recent comprehensive study about CP patterns among CP infants and children attending Alexandria University Children's Hospital over a period of one year (20102011) including: clinical types, topographic types, etiology, and severity.

2. Identifying common associated impairments of CP cases.

3. Comparing our results with other similar studies in Egypt and other developing and developed countries.

\section{OXIDATIVE STRESS IN IRANIAN EPILEPTIC CHILDREN}

doi:10.1136/archdischild-2012-302724.1500

MH Dehghan. Biochemistry, Alborz University of Medical Sceinces, Karaj, Iran

Background Lipid peroxidation is an indicator of free radical metabolism and oxidative stress in human beings and other organisms. Malondialdehyde (MDA), an end product of lipid peroxidation, is a metabolite that can be readily estimated in serum samples. Our objective in this study was to ascertain the variations in alondialdehyde (MDA) in chidren with epilepsy.

Material and Methods This study was carried out in Ardabil and Albroz provinces of Iran after obtaining clearance from the Institutional Ethics Committee. Informed consent was obtained from all the subjects. The quantitative examination of MDA was performed according to standard procedures. The ideal serum level of MDA is below $2.5 \mathrm{nmol} / \mathrm{ml}$.

Results 90 children with confirmed epilepsy (mean age $9.3 \pm 1.5$ ) were included in the study. MDA levels ranged from 1.5 to 3.6 $\mathrm{nmol} / \mathrm{ml}$ (mean level $=2.03 \pm 0.37 \mathrm{nmol} / \mathrm{ml}$ ). Eighty children $(90 \%)$ had MDA levels above the upper limit of normal. Ten patients had levels above $2.85 \mathrm{nmol} / \mathrm{ml}$.

Conclusions This study had shown that the estimation of MDA levels in serum is a convenient method to study lipid peroxidation and thereby oxidative stress in children with epilepsy. Over half of children with epilepsy have excess oxidative stress as indicated by high levels of MDA in the serum. Correlations between MDA level and characteristics of epilepsy, AED therapy, nutritional status and other medical conditions need to be observed in a larger cohort.

\section{QUALITATIVE STUDY OF PSYCHOSOCIAL PROBLEMS AMONG MOTHERS OF CHILDREN WITH CEREBRAL PALSY ATTENDING TERTIARY CARE HOSPITALS IN WESTERN INDIA}

doi:10.1136/archdischild-2012-302724.150

1.2SM Nimbalkar, ${ }^{3} \mathrm{~S}$ Raithatha, ${ }^{1} \mathrm{RA}$ Shah, ${ }^{2} \mathrm{D}$ Antani. 'Department of Pediatrics, Pramukhswami Medical College; ${ }^{2}$ Central Research Services, Charutar Arogya Mandal, ${ }^{3}$ Department of Community Medicine, Pramukhswami Medical College, Anand, India

Background and Aims The parents of children with cerebral palsy face several problems in meeting physical, psychological and social requirements of their children. We explored the psychosocial problems faced by the mothers of children with cerebral palsy in rural and urban settings and compared the same.

Methods Qualitative research design through Focus Group Discussions (FGDs) was utilized. Two FGDs were conducted - one each at rural and urban tertiary level care hospital involving eight and six mothers respectively. Ethical clearance was obtained from Institutional Ethics Committee. The FGDs were conducted by a moderator using an FGD guide and were video recorded. Transcripts were prepared from the video recordings and were analyzed qualitatively.

Results The problems experienced by the mothers were associated with common themes such as - Disturbed social relationships, Physical problems, Moments of Happiness, Worries about future, Need for a special school, Requirement of a support group, Lack of adequate number of trained physiotherapists and Lack of government support. All the parents had children with problems since birth and most had gone to various health care providers for a cure for their child. The participants in the FGD undertaken at the urban based hospital appeared to be poorly adjusted to the problem of the child as compared to the participants in the rural based hospital

Conclusions Current pyscho-social status of the parents should always be considered. Program planners, Hospital administrators and health care providers need to be sensitized about the perceived need of this vulnerable population.

1502 MALFORMATIONS OF CORTICAL DEVELOPMENT AND EPILEPSY: A REPORT OF 13 CASES

doi:10.1136/archdischild-2012-302724.1502 
'S Ben Ameur, 'I Maaloul, 'S Hadj Hmida, 'H Aloulou, 'L Sfaihi, 'I Chabchoub, 'F Kamoun, ${ }^{1} T$ Kamoun, ${ }^{2} \mathrm{Z}$ Mnif, ${ }^{2} \mathrm{M}$ Hachicha. 'Paediatric Department; ${ }^{2}$ Radiology Department, Hedi Chaker Hospital, Sfax, Tunisia

Background and Aims Malformations of cortical development MCDs are increasingly recognized as important causes of epilepsy. The aims of this study is to evaluate the presentation and severity of epilepsy in the differents types of MCDs in children.

Methods Neuroimaging data of patients with epilepsy and MCDs in MRI were evaluated for a period from 2000-2011. The case records were taken from the medical file.

Results We are reporting 13 cases ( 9 boys and 3 girls) of MCDs. The mean age at onset of seizure was 20 months (2 months- 8 years). Psychomotor retardation were present in 5 patients. Craniofacial dysmorphism was noted in 4 cases and microcephaly in 6 cases. Hypotonia and subsequently limb hypertonia were noted in 5 cases. Partial seizure was seen in 5 patients followed by infantile spasms in 3 cases. EEG demonstrated focal epileptiform discharges in 4 cases, and hypsarythmia in 2 cases. Cortical dysplasia was seen in 4 patients, polymicrogyria in 3 patients, lissencephaly in 4 patients and schizencephaly in one patient. Heterotopias were seen in 3 patients in combination with other malformations. Genetic analysis for Miller-Dieker syndrome showed mutations of the LIS1 gene on chromosome 17 in one case. Only 5 Patients had their seizures controlled by antiepileptic drugs ( 2 patients with cortical dysplasia and 3 with polymicrogyria).

Conclusion MR imaging allows the detection and classification of MCDs. An adequate classification of these malformations should help to provide to the family an appropriate counseling both in terms of genetics and outcome.

\section{ACUTE DISSEMINATED ENCEPHALOMYELITIS IN CHILDREN (EIGHT CASES REPORTS)}

doi:10.1136/archdischild-2012-302724.1503

H Ajmi, S Hassayoun, S Mabrouk, N Zouari, S Elkhnissi, J Chemli, S Abroug, K Tlili, A Harbi. Pediatric Department, Sahloul Hospital, Sousse, Tunisia

Background Acute disseminated encephalomyelitis (ADEM) is an immune-mediated inflammatory disorder of the central nervous system, characterized by a widespread demyelination that predominantly involves the white matter of the brain and spinal cord.

We aimed to assess the clinical presentations, neuroimaging, treatment, and outcome of eight children with ADEM.

Methods An 11-years retrospective chart review of children with the diagnosis of ADEM was conducted during the period between 2000 and 2011. The diagnosis of ADEM was carried in front of clinical and radiological signs.

Results Eight cases of ADEM, aged between 9 months and 14 years, were identified. The clinical picture is characterized by multiple symptoms. Prominent findings are seizures in three cases, altered level of consciousness in seven cases and motor system dysfunction in five cases. Brain magnetic resonance imaging evaluations had done in all patients and revealed demyelinated lesions in the cerebral cortex, subcortical white matter, in periventricular white matter, in deep gray matter and in brainstem in all patients. Spinal cord MRI was performed in one case and showed demyelinated lesions in cervical and dorsal etage. All patients were treated with high-dose intravenous methylprednisolone pulse therapy during six days, related by oral corticotherapy. Five of them received also intravenous immunoglobulins. The evolution was favorable in all patients, only one had mild long-term optic neuritis sequelae.

Conclusion Clinical features of ADEM are similar to those of infectious encephalitis. The neuroimaging test of choice to establish the diagnosis is MRI. In most patients, the prognosis is good after treatment.

\section{BDNF AND OXIDATIVE STRESS IN CHILDREN WITH ACUTE LYMPHOBLASTIC LEUKEMIA}

doi:10.1136/archdischild-2012-302724.1504

'MMO Alves, 'PRA Carvalho, 2J Marchesan, 'M Michalowski, 'LLE Daudt, 'L Stertz, 'P Ferrari, 'CS Gama, 'F Kapzinski. 'Universidade Federal do Rio Grande do Sul and Hospital de Clinicas de Porto Alegre, 'Universidade Federal do Rio Grande do Sul, Porto Alegre, Brazil

Background Acute lymphoblastic leukemia (ALL) is the most common malignancy in children, representing nearly one third of all pediatric cancers. Nowadays overall cure rates for children with ALL have reached $90 \%$ due to great improvements in diagnosis and treatment. Unfortunately, the increase in survival rates have been associated with an increase in treatment related toxicity. Survivors may experience late effects from treatment for this condition, such as learning disability and cognitive defects even with no cranial radiation. BDNF levels have been associated with cognitive deficits, among others conditions.

Objectives To evaluate the BDNF levels and oxidative stress in patients with acute lymphoblastic leukemia (ALL).

Designand methods We measured serum concentrations of brain derived neurothrophic factor (BDNF), serumthiobarbituric acid reactive substances (TBARS), serum protein carbonylation, serum IL- 6 and IL-10 before and after 72 hours intratecal methotrexate (MTX). This study was performed on 8 children with ALL during the treatment with BFM protocol and in 40 controls.

Results BDNF levels were lower in ALL patients than in controls $(p<0,001)$. BDNF levels before and after intratecal MTX had not showed significant alterations. Serum protein carbonylation was lower after complete remission.

Conclusion These findings suggested that there is oxidative stress and decreased levels of serum BDNF in patients with acute lymphoblastic leukemia. The treatment may have a protective role in relation to oxidative stress and possible cognitive deficits.

\section{ZINC DEFICIENCY ANEMIA IN SCHOOL CHILDREN}

doi:10.1136/archdischild-2012-302724.1505

HI Atasoy, G Bugdayci. Pediatrics, Abant lzzet Baysal University, Faculty of Medicine, Bolu, Turkey

Background and Aims The objective of this study was to determine the hematologic abnormalities and their potential correlates in school children with zinc deficiency.

Methods 20 parameter hemograms were obtained from the children. Variables that could potentially affect hematological parameters as ferritin, zinc, vitamin B12, and folic acid concentrations were measured in sera of 463 school children. Demographic, anthropometric, biochemical and hematological characteristics of zinc deficient children were compared with those of zinc sufficient children. Associations between potentially related parameters were examined.

Results We showed that zinc deficient and control groups were similar for age and gender ( $p>0.05)$, and zinc deficient children had smaller head circumferences than zinc sufficient children $(p<0.01)$. We also demonstrated lower hemoglobin $(p<0.001)$, hematocrit and red blood cell counts in zinc deficient children, despite similar ferritin levels in both groups. Correlation analysis proved significant relationship between zinc and hemoglobin levels $(p<0.001)$. Linear regression analysis also verified a positive correlation between hemoglobin and head circumference $(p<0.01)$. Logistic regression demonstrated 12 times more odds of anemia in zinc deficient children (OR: 11.9; 95\% CI: 7.0, 20.5).

Conclusions The results implicated that anemia associated with zinc deficiency could not be simply an anemia from iron deficiency but an anemia from deficiency of zinc itself. The results pointed out 